\title{
Vaccinating against human papillomavirus is not associated with risky sexual behaviours among men who have sex with men in Australia
}

\author{
Eric P.F. Chow ${ }^{1,2}$, Ei Aung1 ${ }^{1}$ Marcus Y Chen ${ }^{1,2}$, Catriona S Bradshaw ${ }^{1,2}$, Christopher K Fairley ${ }^{1,2}$ \\ ${ }^{1}$ Melbourne Sexual Health Centre, Alfred Health, Melbourne, Victoria, Australia; \\ ${ }^{2}$ Central Clinical School, Faculty of Medicine, Nursing and Health Sciences, Monash University, Melbourne, Victoria, Australia;
}

\section{BACKGROUND}

- There have been concerns whether vaccinating teenage girls against human papillomavirus (HPV) would lead to risky behaviours.

- A recent meta-analysis has concluded that vaccinating against HPV does not lead to risky behaviours among females ${ }^{1}$.

- However, there has been no studies examining this association among men who have sex with men (MSM).

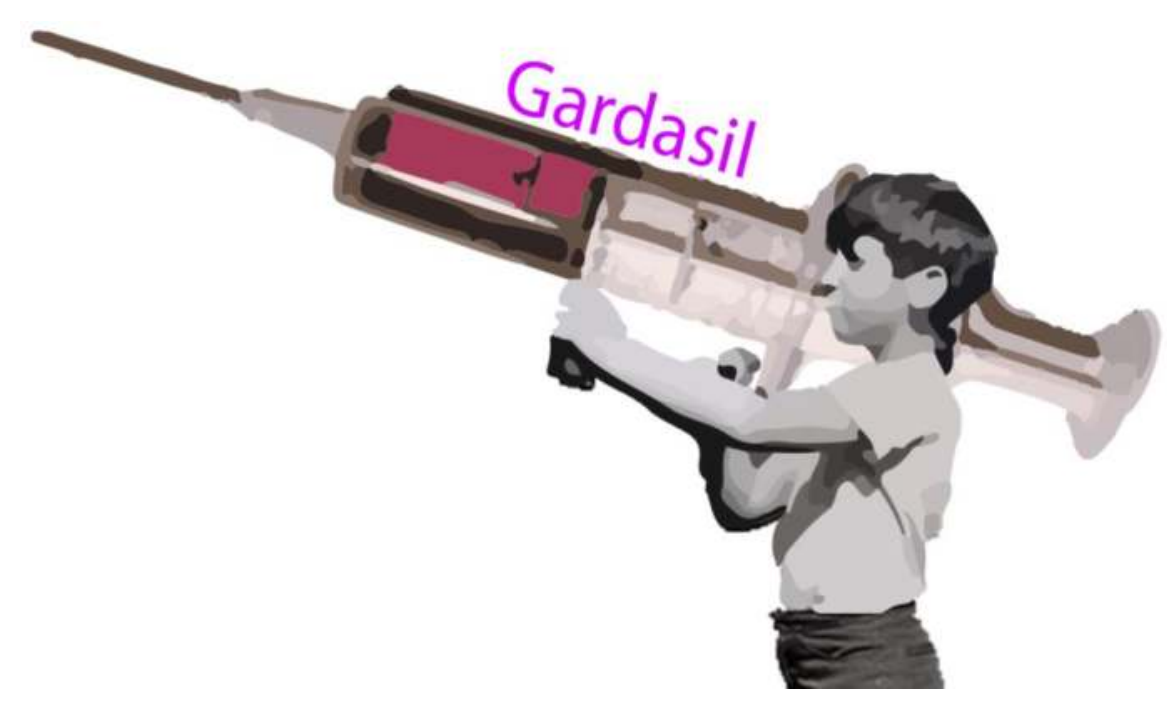

\section{AJM}

- To examine the association between sexual behaviours and HPV vaccination status among men who have sex with men.

\section{METHODS}

- A cross-sectional study was conducted at the Melbourne Sexual Health Centre (MSHC), Victoria, Australia, in 2016.

- Men who reported having any sexual contact with another man (MSM) and aged 16-40 years attending MSHC for their first consultation were asked whether they have been vaccinating against HPV.

- Sexual behaviours data such as number of male partners and condom use in the last 3 and 12 months were collected as part of the routine clinical care.

- Chi-squared and t-test were used to examine the differences in sexual behaviours between vaccinated and unvaccinated MSM.

\section{RESULTS}

- A total of 1,332 MSM aged 16-40 years completed the questionnaire and were included in the analysis.

- The median age of the participant was 27 (IQR 23-31).

- Six percent $(n=81)$ of MSM had been vaccinated against HPV.

- The median number of male partners in the last 3 and 12 months was 2 (IQR 1$5)$ and 5 (2-10), respectively.

- The proportion of men used condoms always in the last 3 and 12 months was $39.2 \%(n=797)$ and $36.5 \%(n=774)$, respectively.

- There were no significant differences in number of partners and always condom use in both last 3 and 12 months between vaccinated and unvaccinated MSM ( $p>0.05$ ) (Table 1)

Table 1. Demographic characteristics and sexual behaviours among men who have sex with men, stratified by HPV vaccination status

\begin{tabular}{|c|c|c|c|}
\hline Characteristics & $\begin{array}{c}\text { Vaccinated } \\
(\mathrm{N}=81)\end{array}$ & $\begin{array}{l}\text { Unvaccinated } \\
\qquad(\mathrm{N}=1251)\end{array}$ & P value \\
\hline Age, mean $\pm S D$ & $26.8 \pm 0.7$ & $27.7 \pm 0.1$ & $0.151^{\wedge}$ \\
\hline Country of birth, $n(\%)$ & & & $0.562^{\Phi}$ \\
\hline Australia & $36(44.4 \%)$ & $515(41.2 \%)$ & \\
\hline Overseas & $45(55.6 \%)$ & $736(58.8 \%)$ & \\
\hline
\end{tabular}

last 3 months, mean $\pm S D$

$4.0 \pm 0.6$

$4.0 \pm 0.2$

$0.967^{\wedge}$

Condom use with male partners

in the last 3 months, $\mathrm{n}(\%)$

$0.566^{\Phi}$
Always
Not always $28(36.8 \%)$
$48(63.2 \%)$ $462(40.2 \%)$ $48(63.2 \%) \quad 688(59.8 \%)$

Number of male partners in the last 12 months, mean $\pm S D$

$\begin{array}{ll}9.8 \pm 2.3 & 8.8 \pm 0.4\end{array}$

$0.553^{\wedge}$

Condom use with male partners in the last 12 months, $\mathrm{n}(\%)$
Always
Not always
$24(31.2 \%)$
$461(38.5 \%)$
$53(68.8 \%)$
$737(61.5 \%)$

${ }^{\wedge}$ t-test; ${ }^{\Phi}$ Chi-squared test

\section{CONCLUSIONS}

- Vaccinating against HPV is not associated with increased sexual activity and condomless anal sex practice among MSM, particularly among sexually-active men attending a sexual health service

\section{REFERENCE}

1. Kasting ML et al. Tempest in a teapot: A systematic review of HPV vaccination and risk compensation research. Human Vaccines \& Immunotherapeutics. 2016; 12: 1435-1450. 